\title{
La Asociación Parque Sí en Chamberí y la participación de los vecinos en la definición y uso del Parque sobre el Tercer Depósito del CYII en Chamberí
}

\author{
"Asociación Parque Sí" in Chamberí and the participation of \\ neighbors on the definition and use of "Parque sobre el Tercer \\ Depósito" of CYII in Chamberí
}

\author{
Mercedes Arce Sainz ${ }^{1}$ \\ Fecha de recepción: 02-02-2021 - Fecha de aceptación: 03-03-2021 \\ Hábitat y Sociedad (ISSN 2173-125X), n. ${ }^{\circ}$ 14, noviembre de 2021, pp. 327-337. \\ https://doi.org/10.12795/HabitatySociedad.2021.i14.17
}

\section{Summary}

There are not many cases in which an urban movement, rising from the bottom up, manages to achieve its objectives through a continuous, creative and diverse struggle, with the complicity and support of many residents.

The decision of illegally changing the use of a public park into a golf course materialized in the creation of the association Parque Sí en Chamberí, which, in time, used all the means at its disposal (legal, participatory, cultural, support in the street) to topple this decision and, in the meantime, created a social associative network which strengthened the district's social capital.

\section{Key words}

Public parks; Residents' association; Citizen participation; Right to the city; bottom-up processes

\section{Resumen}

No son muchos los casos en que un movimiento urbano que surge de abajo arriba consigue, a través de una lucha continuada, creativa, diversa, incluyendo la complicidad y el apoyo de muchos vecinos, lograr sus objetivos.

La decisión de cambiar ilegalmente el uso de un parque público, por una instalación de golf se concretó en la formación de la Asociación Parque Sí en Chamberí, que al tiempo que utilizó todas las vías en su mano (jurídicas, participativas, de apoyo en la calle) para cambiar esta decisión, y a la vez fue creando un tejido asociativo que reforzó el capital social del distrito.

\section{Palabras clave}

Parques públicos; Asociaciones vecinales; Participación ciudadana; Derecho a la ciudad; Procesos de abajo arriba 


\section{Introducción}

Este artículo explica la historia de un movimiento vecinal que consigue sus objetivos. El proceso ha supuesto un enorme esfuerzo de energía y solidaridad, empleando la inteligencia colectiva, el tiempo y la voluntad de mucha gente comprometida, con diferentes niveles de implicación, en un objetivo de mejora de la ciudad de abajo arriba.

El objetivo de este relato es compartir esta experiencia de acción colectiva para que pueda servir de referencia en otras situaciones similares en el marco de reflexión sobre un futuro en el que el diseño y gestión de la ciudad sean mucho más democráticos y dirigidos en beneficio de las personas.

Chamberí es un distrito de Madrid con 140866 habitantes (2019), (Figura 1) con una densidad de 301,05 Hab./Ha. y con una edad media de más de 46 años. El índice de dependencia es de 53,2 \%, fren-

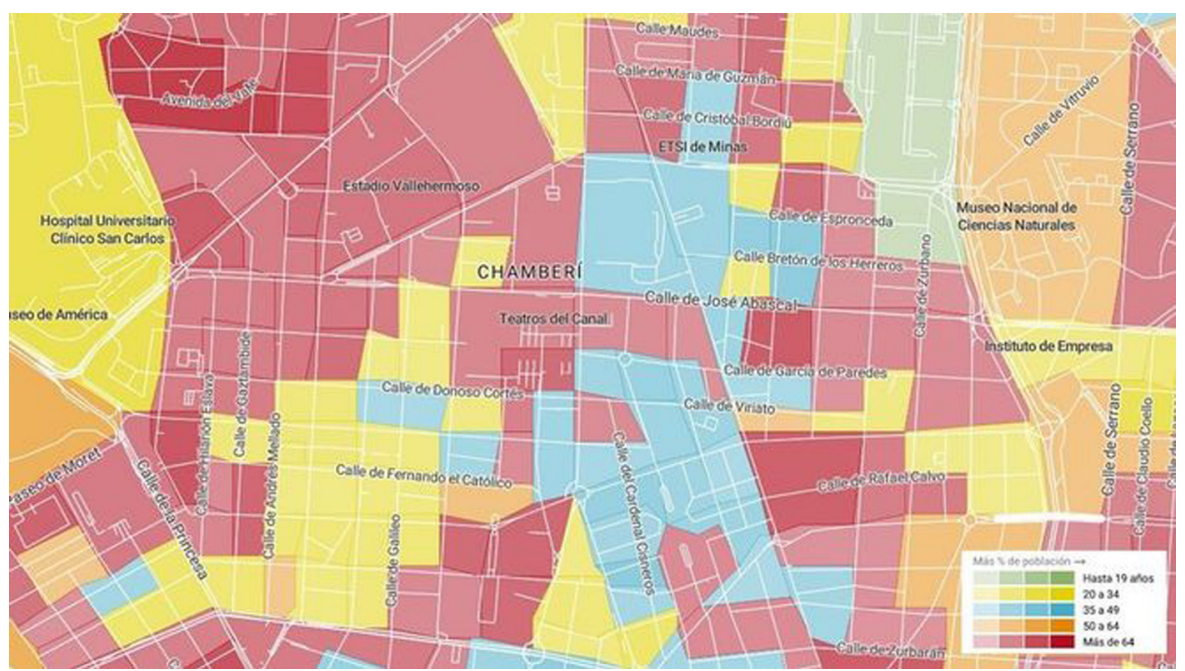
te al $51,6 \%$ de todo Madrid. El índice de envejecimiento de $225,1 \%$ supera ampliamente el de todo Madrid, de un $143,5 \%$. Por tanto, es un distrito que necesita espacios verdes para hacer posible tanto el envejecimiento activo de sus mayores como la crianza de las familias que están renovando estos barrios.

Por poner en cifras esta carencia de zonas verdes de libre acceso público, recogemos los indicadores de zonas verdes: Chamberí (Figura 2) cuenta con menos de $1 \mathrm{~m}^{2}$ de zona verde por residente, lejos de los 10$15 \mathrm{~m}^{2} / \mathrm{hab}$. de zonas verdes que recomienda la OMS, de los $5,88 \mathrm{~m}^{2} /$

Figura 1: Grupos de población en Chamberí en 2019. Fuente: Captura a partir de eldiario.es. hab. de la Ley del suelo vigente de la CAM o de los cerca de $20 \mathrm{~m}^{2} /$ hab. de media en Madrid.

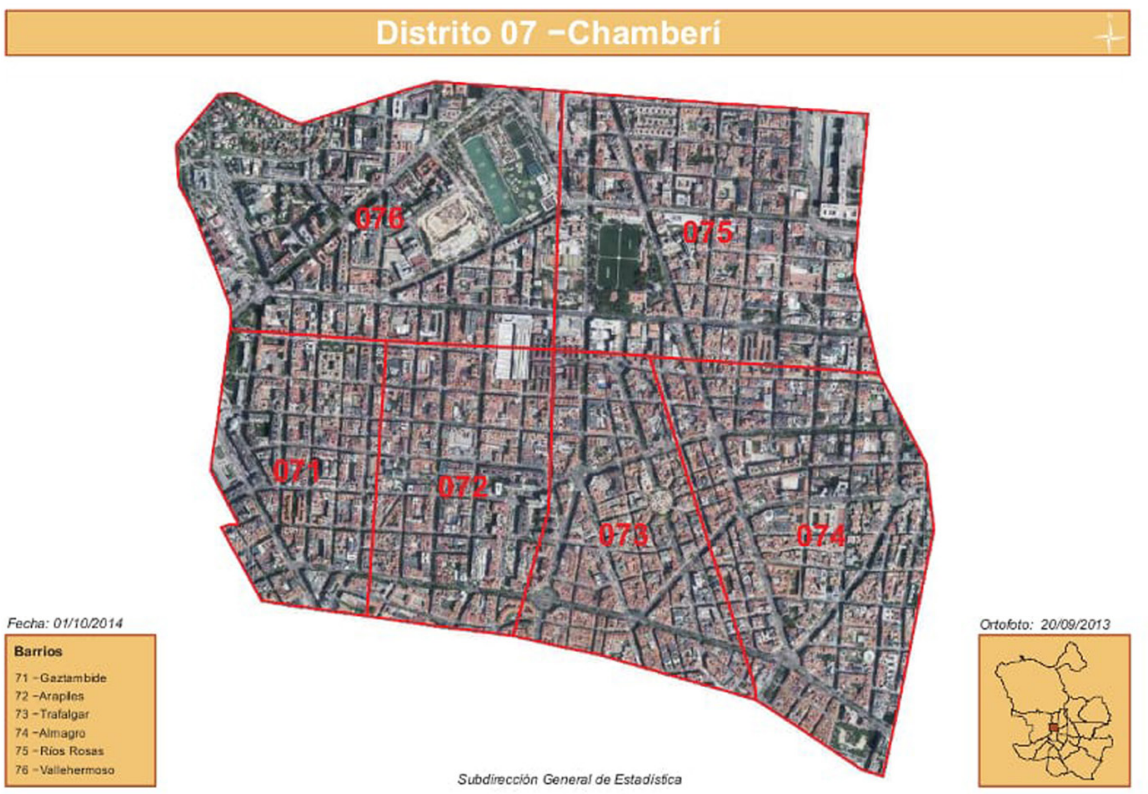

Figura 2: El distrito de Chamberí en la almendra central de la ciudad de $\mathrm{Ma}^{-}$ drid. Fuente: Ayuntamiento de Madrid. 
En Chamberí surge un movimiento de abajo arriba que se caracteriza por las siguientes formas de actuar:

- Ser más propositivo que crítico.

- Utilizar todas las vías de acción posibles.

- La paciencia y la tenacidad de seguir en la misma línea durante más de quince años.

- La capacidad de presentar propuestas alternativas surgidas desde la organización de espacios de participación.

Tras esta primera reflexión, el artículo explica, desde el testimonio en primera persona de sus protagonistas, cómo se ha desarrollado este largo proceso a lo largo de estos años.

\section{Descripción del proceso}

"Nunca dudes de la capacidad de un grupo de ciudadanos pensantes y comprometidos, para cambiar el mundo. De hecho, son los únicos que lo han logrado" (Margaret Mead). Este lema es el que ha guiado a nuestra Asociación Parque SÍ en Chamberí (a partir de ahora Parque SÍ) todos estos años. Las vecinas y vecinos de Chamberí no tenemos ningún parque grande, ni espacios adecuados para jugar o disfrutar de la naturaleza. Es el distrito con menos zonas verdes de Madrid. Siempre mirábamos con sentimiento de frustración el gran espacio libre, pero inaccesible, propiedad del Canal de Isabel II (en adelante CYII), la empresa pública más importante de la Comunidad de Madrid, que gestiona el agua en Madrid y posee cuatro grandes depósitos en nuestro distrito. Los vecinos pedíamos desde hace décadas la apertura al público de las amplias zonas verdes que tienen en el distrito.

En 2002, la Comunidad de Madrid firmó un convenio con el Ayuntamiento de Madrid en el que, a cambio de conceder una importante edificabilidad al CYII en los diversos terrenos que tiene en Chamberí, se acondicionaría un parque en los terrenos del Tercer depósito y se abriría el Segundo depósito.

Nos prometieron un gran parque como se publicó en la prensa (Figura 3) y en las vallas publicitarias ubicadas en el propio Parque. Y nos lo creímos.

Por eso, cuando a principios de 2006 se iniciaron las obras, todos pensamos que, ipor fin!, estaban construyendo ese gran parque, sin darnos cuenta, en ese momento, de que se trataba de un proyecto dis-

COMUNIDAD DE MADRID AYUNTAMIENTO DEMADRID CERCANIAS METRO EMT MADRID CENTRAL ÚLTIMAS NOTIIIAS

Te quedan 9 artículos gratis este mes

\section{Chamberí tendrá sobre los depósitos del Canal un parque de 118.000 metros con un helipuerto en el centro}

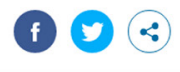

Figura 3: Artículo de prensa sobre el Futuro parque 15 de abril de 2003. Fuente: El País. 
Figura 4: Manifestación pro-parque el 18 de diciembre de 2006. Fuente: Asociación Parque Sí en Chamberí.
Figura 5: Grupo del taller y maquetas realizadas por niños y niñas en el primer taller participativo. Fuente: Asociación Parque Sí en Chamberí. tinto y que nos estaban engañando. Todo ello se realizó sin información pública y sin transparencia alguna, motivando el inicio de las protestas vecinales.

\section{Participación y Movilización Vecinal}

Los vecinos empezamos a ver cómo se levantaban enormes torres y redes metálicas para delimitar grandes espacios en los que se colocaba césped artificial y palmeras de plástico. El asombro y la indignación iba en aumento, a la par que parecía que no podría hacerse nada ante estos hechos consumados.

Algunos vecinos empezamos a recabar información sobre el nuevo proyecto. Fue en ese momento cuando entendimos el alcance de la operación. Nuestro parque estaba siendo sustituido por una instalación para la iniciación y práctica de golf que ocupaba $45000 \mathrm{~m}^{2}$ del total de los $91000 \mathrm{~m}^{2}$ del nuevo Parque.

Poco a poco se organizó un gran movimiento vecinal en el barrio (Figura 4) con diversas acciones y numerosos apoyos. Y a ello se sumaron informes desfavorables a este proyecto por parte de la CPPHAN
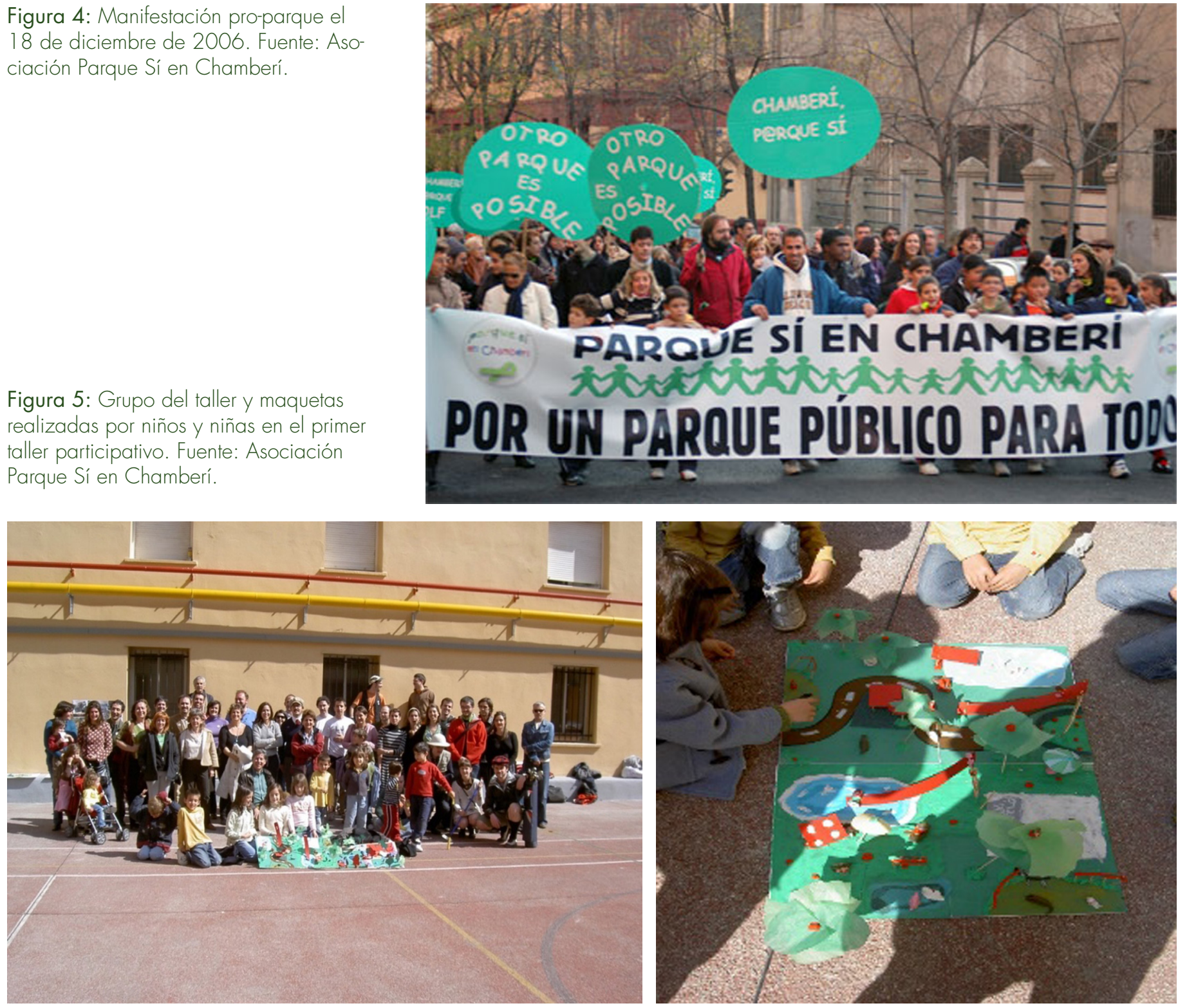
y del Departamento de Urbanismo de la ETSAM. Finalmente, en octubre de 2006, el Ayuntamiento ordenó la suspensión de las obras.

Parque Sí se constituyó como asociación en 2007 y, desde entonces, se han desarrollado acciones legales y de divulgación, ${ }^{2}$ manifestaciones, recogida de firmas, pancartas y lazos verdes en los balcones, mesas en la calle, fiestas para recaudar fondos, talleres para definir un proyecto del Parque en el III Depósito del CYII.

En marzo de 2007 organizamos el primer taller participativo (Figuras 5 y 6) para definir cómo podría ser el parque, con charlas, talleres de vecinos y también taller de deseos con maquetas infantiles.

\section{Participación y acciones legales}

El 18 de enero de 2007 el Consejo de Gobierno de la Comunidad de Madrid, presidido por Esperanza Aguirre, declaró el golf de "interés general". El 25 de enero de 2007, la AAVV el Organillo de Chamberí, con el apoyo de Parque SÍ, interpuso un recurso de reposición contra el Acuerdo de dicha declaración de "interés general". El 12 de diciembre de 2012 el Tribunal Superior anula la declaración de interés general de la Comunidad de Madrid y esta, para legitimar el golf, elabora un Plan Especial.

El 8 de noviembre de 2013, Parque Sí, interpuso un contencioso administrativo contra ese Plan Especial. Y en julio de 2016, el TSJM dictó la sentencia que declaraba nulo este Plan Especial. Diez años habían transcurrido desde el comienzo de la construcción irregular de las instalaciones de golf en el III Depósito del CYII de Madrid.

Entre los diferentes motivos de nulidad a los que aludía la sentencia, destacamos dos:

1) La ausencia de motivación de la implantación de una instalación de golf sobre unos terrenos destinados a zona verde.

2) La desviación de poder cometida por el CYII y la Comunidad de Madrid.

\section{Participación y nueva etapa: diálogo para el diseño del Parque}

El 6 de octubre de 2016, tuvimos la primera reunión con el CYII a la que acudieron Ángel Garrido, Vicepresidente de la Comunidad de Madrid y Presidente del CYII, Enrique Ruiz Escudero, Viceconsejero de Presidencia y Justicia y Rafael Prieto Martín, el nuevo Director del CYII, con el que a partir de ese momento estuvimos negociando. Y por parte de Parque Sí, acudimos 8 personas incluido nuestro abogado, Jaime Doreste, al que siempre estaremos agradecidos por su trabajo en pro de recuperar el parque. Esta fue la primera de las numerosas reuniones, cerca de treinta, que hemos mantenido desde entonces representantes de nuestra Asociación con abogados, arquitectos y técnicos del CYII.
Figura 6: Documento resumen del taller 'Un buen parque para Chamberi'. Fuente: Parque Sí en Chamberí.

2 Como ejemplo de la amplia difusión de este problema, el programa de televisión "Caiga quien Caiga" dedicó un reportaje al Campo de Golf en el Parque del Tercer Depósito el CYII en Chamberí, febrero de 2007 https://www.parquesienchamberi.org/archives/603. 


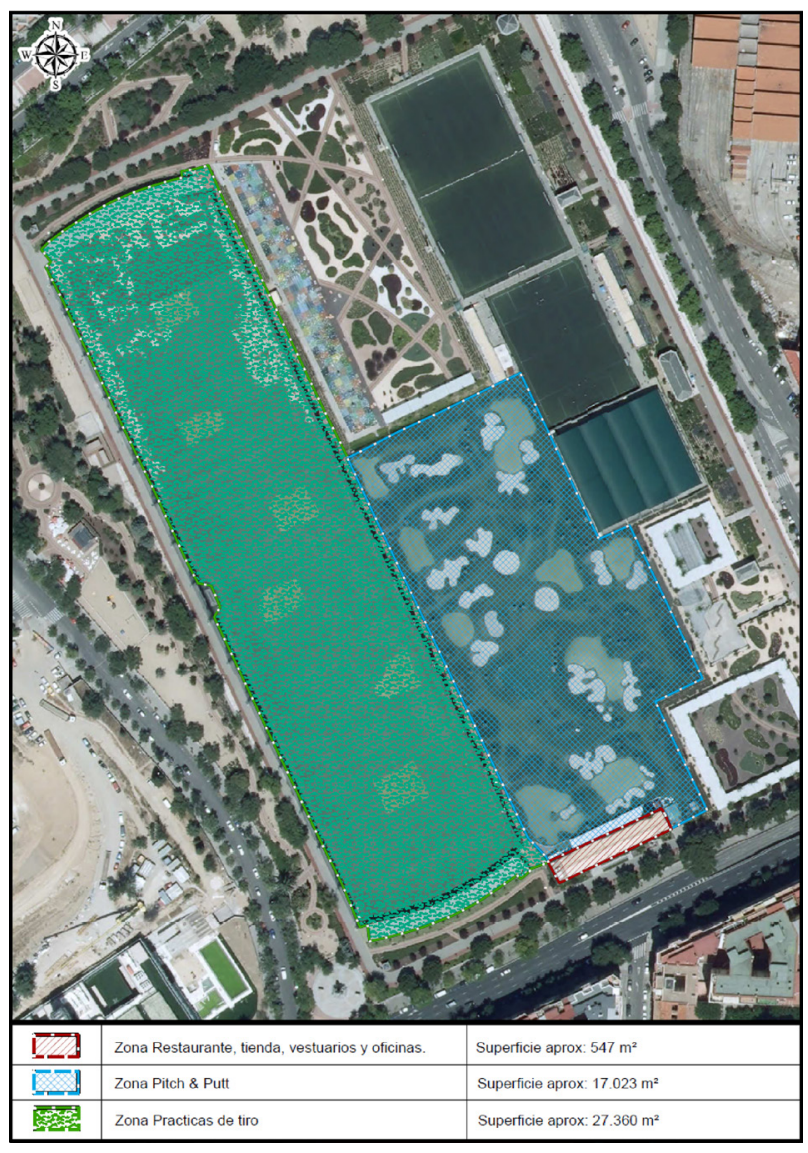

Figura 7: Plano proporcionado por el Canal en la primera reunión, en octubre de 2016. Fuente: Canal Isabel II.

Figura 8: Documento de ideas clave del taller de participación mayo 2017. Fuente: Asociación Parque Sí en Chamberí
En todas las reuniones se han planteado las siguientes propuestas ante el Canal:

1) La apertura completa del Segundo Depósito: ya hemos conseguido $7200 \mathrm{~m}^{2}$.

2) La restauración de la Fuente en honor al Río Lozoya, hemos conseguido que tenga agua.

3) La apertura de la sala de exposiciones en el Depósito Uno, para sede de un Museo del CYII.

En esta primera reunión, solicitamos realizar un taller participativo que recogiera las necesidades de nuestro distrito y los planos del parque (Figura 7), con el fin de situarlas. Ante la negativa del CYII a hacer un proceso participativo, Parque Sí, decidió organizarlo.

El taller se realizó el 14 de enero de 2017 con el objetivo de definir un proyecto que transformara los $45000 \mathrm{~m}^{2}$ que ocupaban las instalaciones de golf, tratando de resolver, al menos parcialmente, los déficits del distrito en materia de zonas verdes, así como de atender a las necesidades y deseos de la mayor parte de la ciudadanía.

Contratamos a tres empresas especializadas en urbanismo y participación: n'Undo, Red de Saberes y La Locomotora. Los resultados del taller se concretaron en un Informe sobre el taller, un programa de necesidades y un resumen de la gran cantidad de ideas recogidas en el mismo.

Parque Sí, presentó las conclusiones del taller al CYII en un documento que se llamó Diez ideas-clave para el diseño del nuevo Parque del Canal (Figura 8).

El CYII acepta el documento y en julio 2017, el Área de Arquitectura y Urbanismo del CYII nos presentó el documento Programa de necesidades, nuevo parque Canal Tercer Depósito, sobre el III Depósito del CYII, en

\section{PROGRAMA DE NECESIDADES PARA EL PARQUE DE CANAL}

RESULTADO DE UN PROCESO DE DIALOGO VECINAL CONTINUADO DURANTE 10 AÑOS, CON VARIOS PROCESOS PARTICIPATIVOS Y DEBATES

\section{A. 10 IDEAS CLAVE PARA EL DISEÑO Y CONFIGURACIÓN DEL PARQUE}

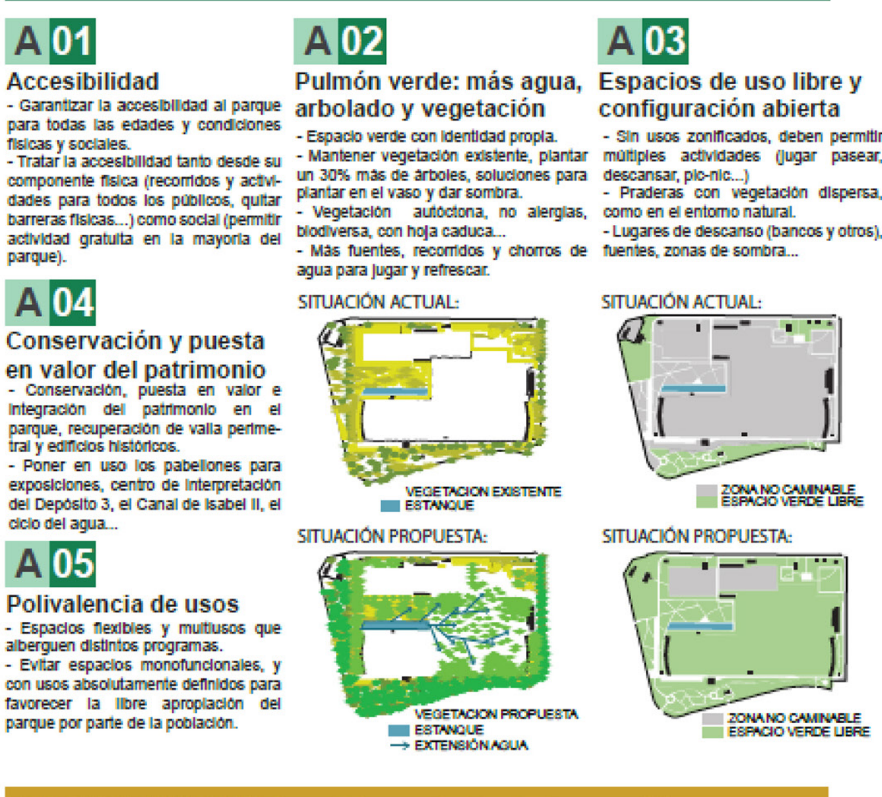

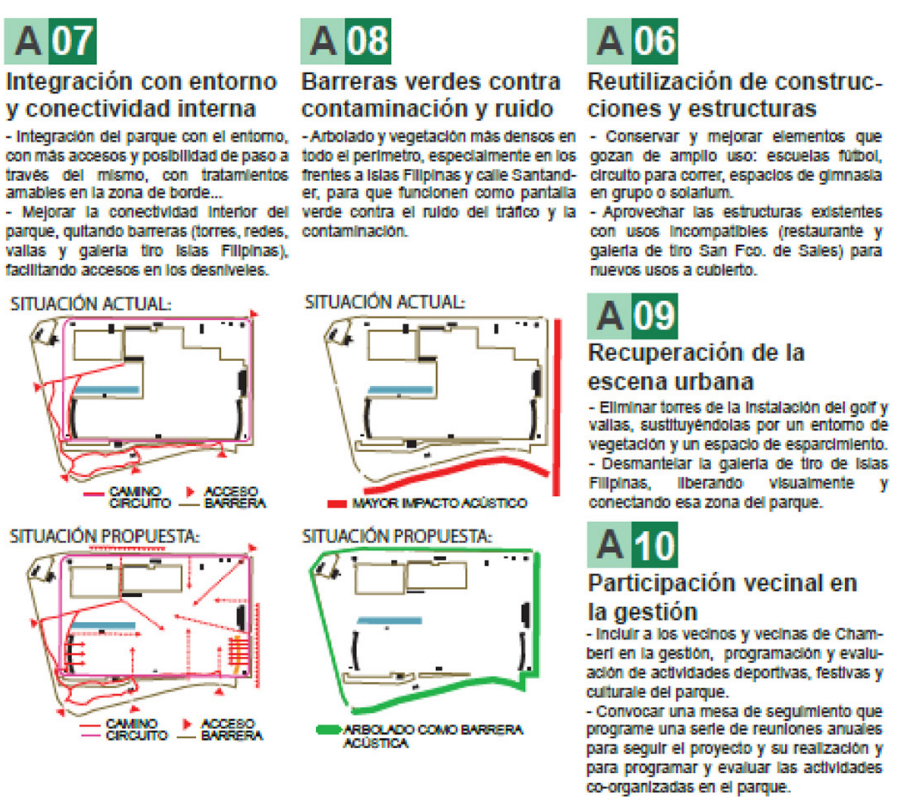


la Avda. Filipinas de Madrid y el plano del futuro parque (Figuras 9 y 10). En el mismo, se recogían las ideas presentadas en nuestros documentos Diez ideas-clave para el diseño del nuevo Parque del Canal, las conclusiones del taller participativo de enero 2017 y algunas determinaciones técnicas presentadas.

El junio de 2018, el CYII solicitó la licencia ante el Ayuntamiento para realizar el nuevo parque con el proyecto diseñado por los vecinos.

Así pues, finalmente, habían aceptado a grandes rasgos las ideas que habían surgido como resultado de tantos años de contacto con los vecinos. Pero ahora quedaba por conseguir lo más difícil, aumentar el número de árboles, para cumplir el objetivo de que este Parque fuera un auténtico pulmón verde para Chamberí.

El logro de conseguir el mayor número de árboles se tradujo en muchas reuniones con los dos interlocutores nombrados por el CYII, personas con las que hemos dialogado estos años y a los que también les agradecemos todo el trabajo realizado.

En junio de 2018, después de haber consultado a ingenieros agrónomos, paisajistas y especialistas en jardinería, presentamos al CYII un documento (Figura 11)

Este documento, redactado por Ramón López Lucio, Doctor Arquitecto-Urbanista, miembro de Parque Sí, en el que se recomiendan los trazados y las especies de árboles que se deben plantar (Figura 12), las técnicas de plantación encima del depósito soterrado, naturalizar los

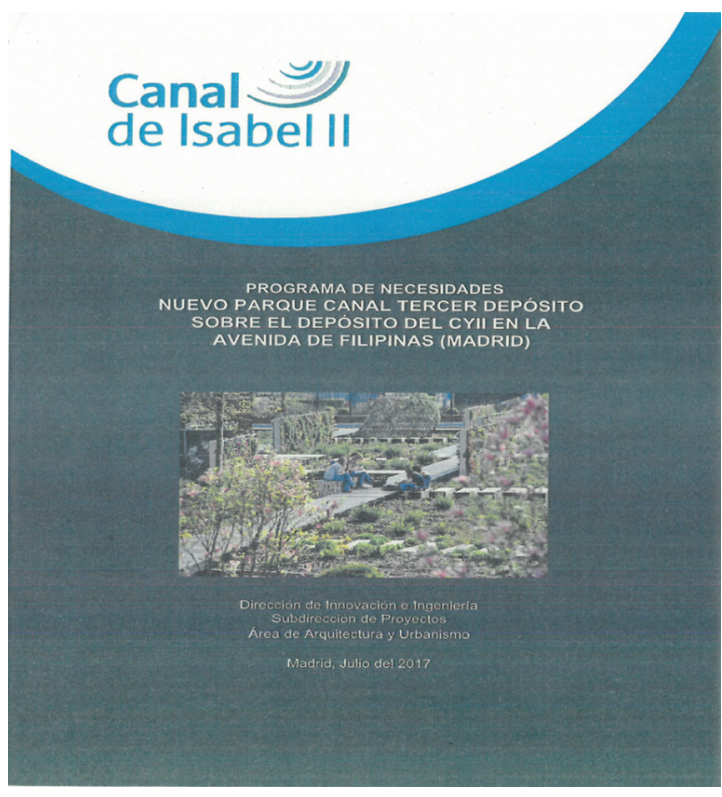

Figura 9: Documento de programa de necesidades del CYII, julio 2017. Fuente: Canal Isabel II.

Figura 10: Plano futuro parque presentado por Canal 2017. Fuente: Canal de Isabel II.

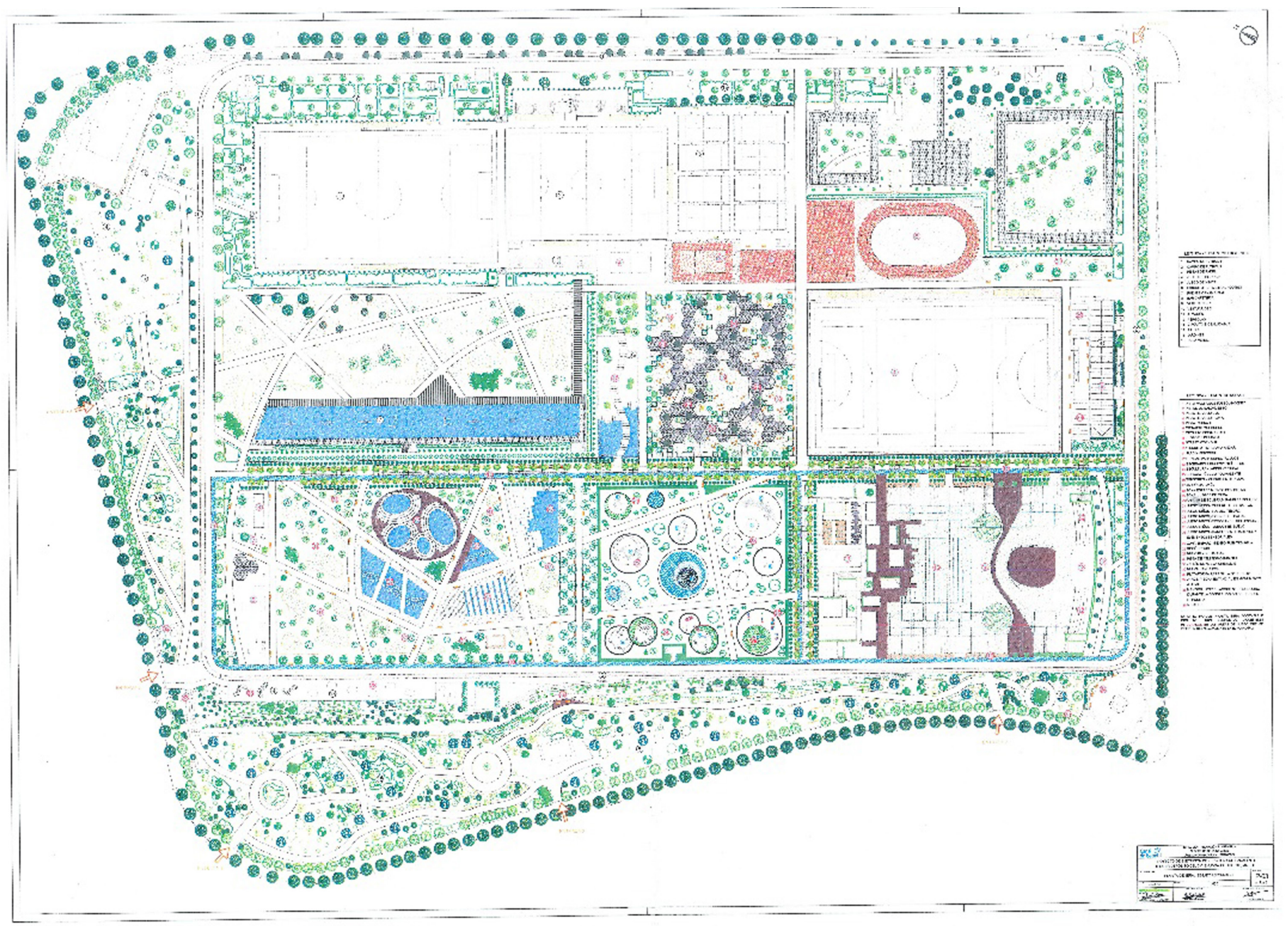


Figura 11: Informe presentado a CYII, junio 2018 . Fuente: Asociación Parque Sí en Chamberí. taludes y muros de hormigón existentes y las nuevas zonas que se van a crear: pistas de correr, plaza cívica, duplicar las zonas de agua.

\section{SUGERENCIAS DE DISEÑO, TÉCNICAS DE PLANTACIÓN y SELECCIÓN DE ESPECIES PARA LA FASE FINAL DEL PROYECTO DE NUEVO PARQUE SOBRE EL TERCER DEPÓSITO DEL CANAL DE ISABEL II y MEJORAS EN EL ACTUAL PARQUE DE SANTANDER (*).}

Junio 2018

\section{INTRODUCCIÓN}

Después de las diferentes reuniones celebradas durante los últimos meses entre responsables y técnicos del CANAL DE ISABEL II con técnicos y personas representativas de la plataforma PARQUE SÍ EN CHAMBERÍ así como de otros grupos y asociaciones del Distrito y con diferentes especialistas en jardinería y diseño de espacios verdes urbanos (Luciano Labajos, José Molina, Isabela Velázquez, entre

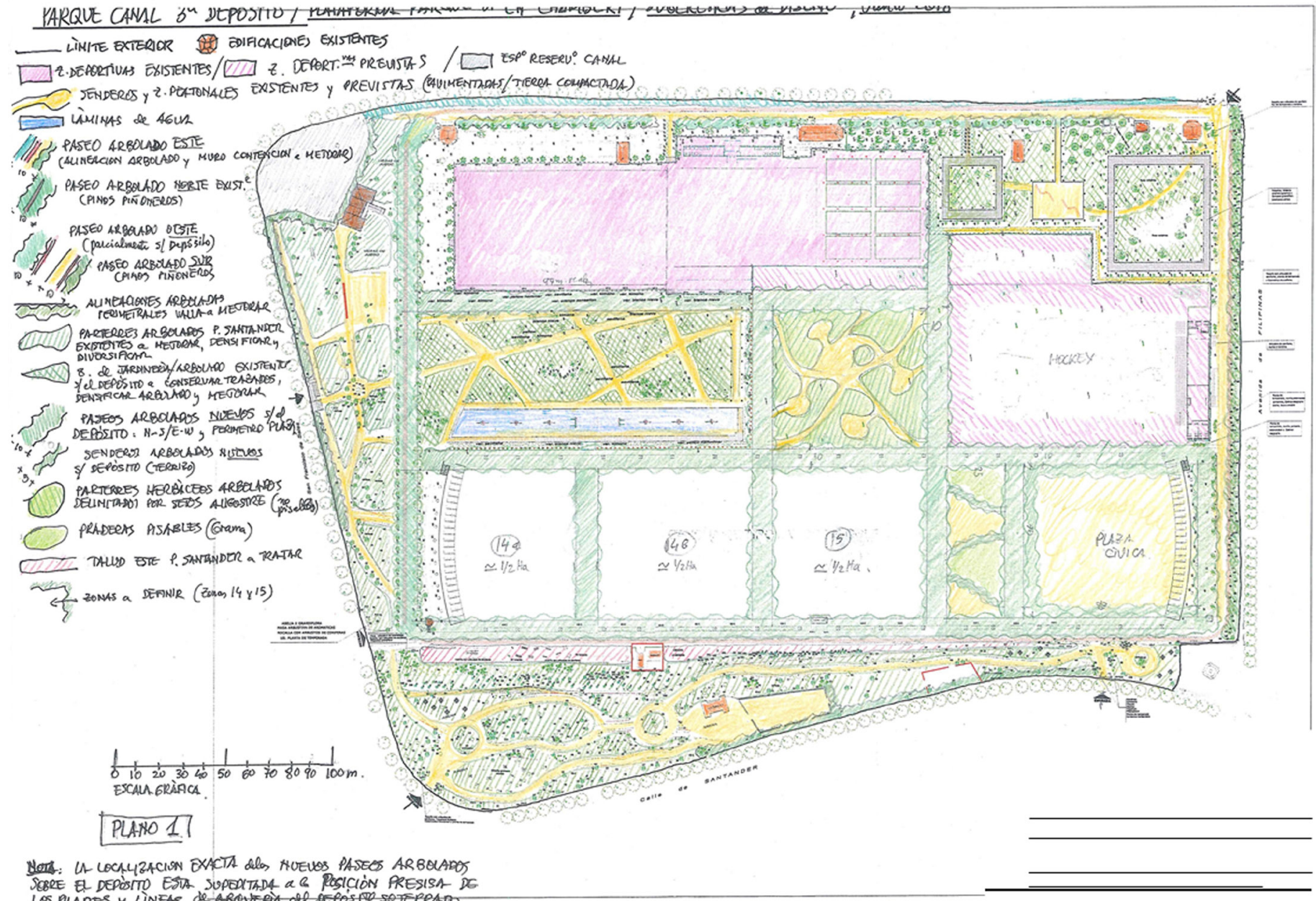

Figura 12: Plano sugerencias nuevo parque. Fuente: Asociación Parque Sí en Chamberí.

\section{La Asociación Parque Sí en Chamberí y el fomento de la participación vecinal en el distrito de Chamberí}

Antes de finalizar este artículo no queremos dejar de señalar la importancia que nuestra Asociación ha tenido en nuestro distrito. Parque Sí, se constituyó para reivindicar un parque para todos, sin embargo, el largo proceso hasta conseguir el objetivo ha provocado que Parque Sí se implique en muchas otras iniciativas. En este sentido, hemos colaborado y seguimos colaborando con otras asociaciones y entidades sociales, para formular una visión de futuro de este distrito. 
Periódicamente hemos organizado eventos participativos, paseos críticos por el distrito, reuniones y, entre otras iniciativas de éxito que nos han ayudado a mantener el contacto con los vecinos, es preciso mencionar las fiestas en dos salas muy conocidas en Madrid, Clamores y Galileo Galilei, a cuyos propietarios agradecemos su apoyo

De entre las fiestas realizadas, queremos destacar la que organizamos el 18 de febrero de 2013, en la que invitamos a participar a todas las asociaciones del distrito que conocíamos. ¡El resultado fue genial! pues surgió un grupo de trabajo que lo denominamos "Chamberí se mueve" (Figura 13). Este grupo de trabajo ha seguido reuniéndose y comentando lo que iba ocurriendo en el distrito y lo que desde cada Asociación se iba planteando. $\mathrm{Y}$ ha sido el precedente de la actual Casa de la Cultura de Chamberí en la que hemos participado muy activamente.

En 2017 nos reunimos dieciséis asociaciones y colectivos de Chamberí y presentamos un proyecto, al que llamamos Casa de Cultura y Participación Ciudadana de Chamberi (Figura 14) Este proyecto salió elegido y nuestra asociación, junto a otras, recibió la cesión de uso del local municipal. La Casa de la Cultura de Chamberí, espacio ciudadano, auto gestionado por 24 colectivos donde se ha fomentado el asociacionismo, la vertebración social y la participación vecinal.

Entre las actividades de participación ciudadana, que hemos orga-
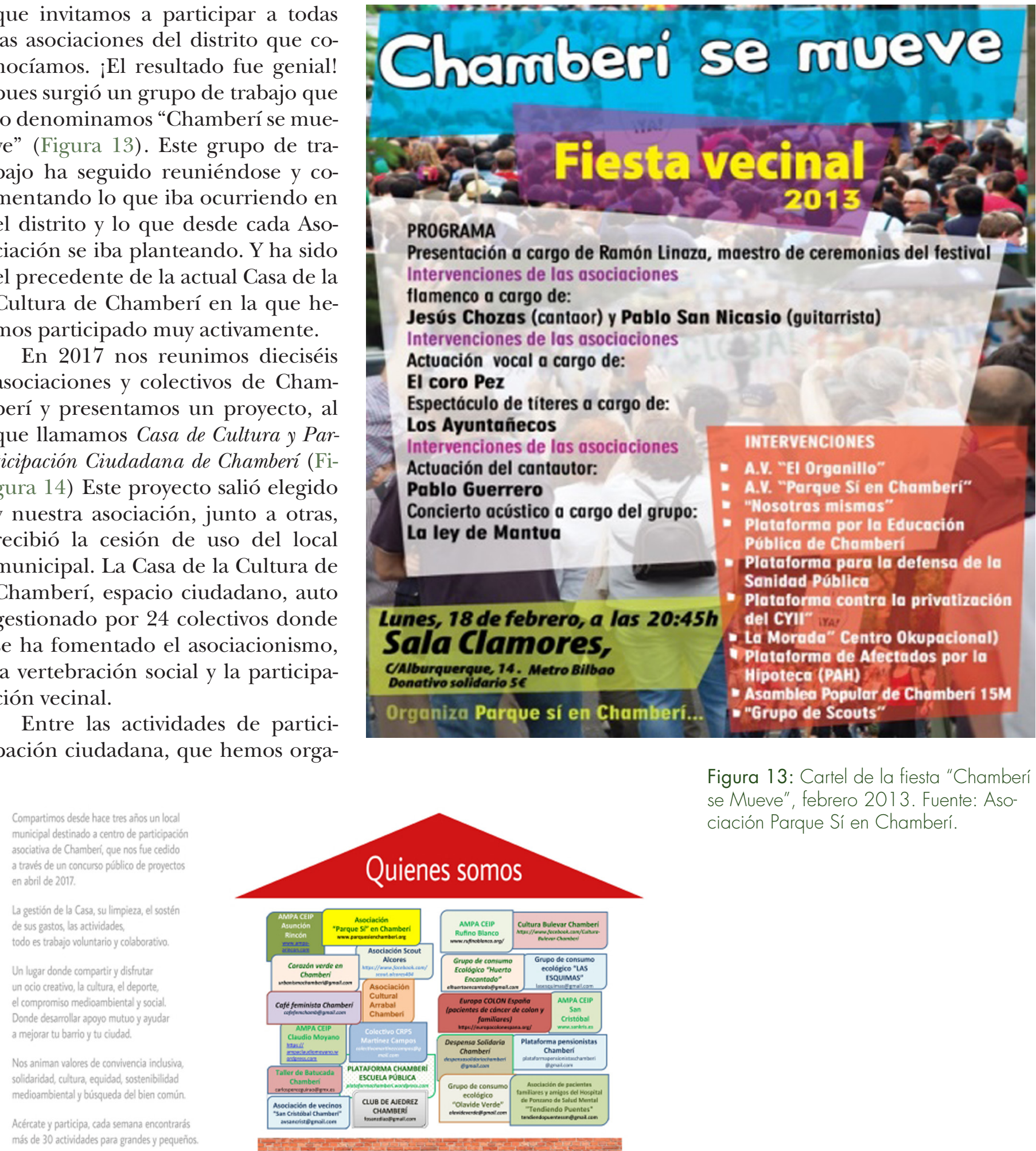

Figura 13: Cartel de la fiesta "Chamberí se Mueve", febrero 2013. Fuente: Asociación Parque Sí en Chamberí.
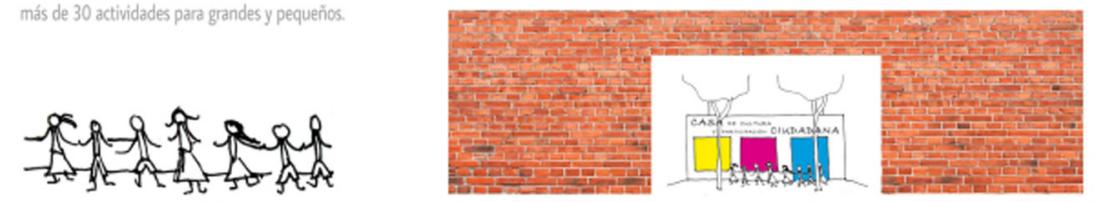

Figura 14: díptico Casa de Cultura ya participación de Chamberí. Fuente: Asociación Parque Sí en Chamberí. 


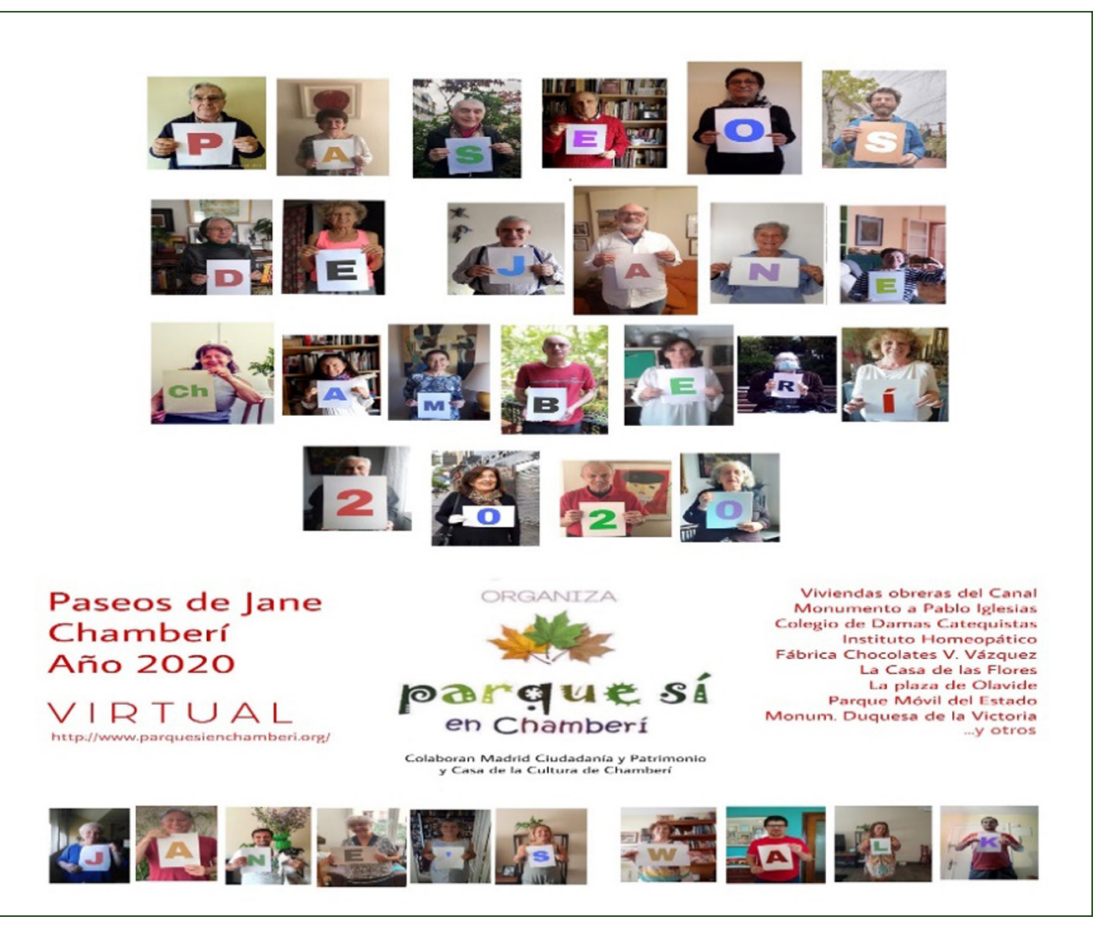

Figura 15: Paseo de Jane virtual, mayo 2020. Fuente: Asociación Parque Sí en Chamberí. nizado anualmente desde 2015, la que ha convocado a más personas y mayor complicidad ha generado entre los participantes, ha sido los "Paseos de Jane Chamberî" (Figura 15).

En nuestra Asociación, desde que empezamos a finales del 2006, han primado valores como la constancia, la perseverancia, la colaboración con unos y otros, el reconocimiento de las diferentes opiniones, el diálogo, el respeto, el trabajo en equipo..., que han sido la base para alcanzar nuestro objetivo, un objetivo claro y concreto: un gran Parque para Chamberí. También la alegría, la celebración, el agradecimiento, la fiesta, el teatro y el encuentro han sido factores clave.

Nos parece que nuestra experiencia es un referente de cómo impulsar la vertebración de la sociedad civil en nuestra ciudad junto a las instituciones locales.

\section{Agradecimientos}

A todas las vecinas y vecinos de Chamberí; a todas las personas, que son muchas, que han colaborado en estos años de lucha ciudadana. Y por supuesto a nuestros compañeros Fernando Casado, Ilda Fava, Juan Manuel Villar, y Pepe Nieto, que nos han dejado, que la tierra les sea leve. 


\section{Referencias bibliográficas y audiovisuales}

Asociación Parque Sí en Chamberí. https://www.parquesienchamberi.org/.

Asociación Casa de la Cultura y de la Participación de Chamberí. https://casaculturachamberi.com/.

Atresmedia Televisión Globomedia (2017, 28 de junio). Los vecinos reclaman un Parque. El Intermedio. Recuperado el 16 de marzo de 2021 de: https:/ / www. lasexta.com/programas/el-intermedio/gonzo/carmen-ochoa-vecina-de-chamberi-presentamos-unaquerella-contra-gonzalez-clavada-a-la-que-le-llevo-a-lacarcel_20170628595413fb0cf26ceeda3b4061.html.

Basurama (2007, 17 de marzo).Madrid ama el Golf'. Recuperado el 16 de marzo de 2021 de: https:/ / www.youtube.com/ watch?v=mZaIHqwoYYU\&feature $=$ emb_logo.

La Locomotora (2017, 19 de enero). Parque Canal (taller de participación infantil: El parque que queremos). Recuperado el 16 de marzo de 2021 de: https:/ / www.parquesienchamberi.org/archives/679.

López Lucio, Ramón et al., (2018). Sugerencias de diseno, técnicas de plantación y selección de especies para la fase final del proyecto de nuevo parque sobre el Tercer Depósito del CYII y mejorasen el actual Parque de Santander. Recuperado el 24 de mayo de 2021 de: https:// www.parquesienchamberi.org/archives/1134.

López Lucio, Ramón (2010). Dictamen urbanístico sobre el supuesto carácter de "interés general" de las instalaciones de golf implantadas sobre el 3. ${ }^{\circ}$ depósito del Canal de Isabel II."Recuperado el 16 de marzo 2021 de: https://www.parquesienchamberi.org/wp-content/ uploads/2013/01/CANAL-GOLF-Dictamen-urbanistico.2010.pdf.

Madrid Actual, Cine y TV (2021, 24 mayo). Un documental recuerda la lucha vecinal que consiguió un parque para Chamberí. Recuperado el 24 de mayo de 2021 de: https: / / www.madridactual.es/7769214un-documental-recuerda-la-lucha-vecinal-que-consiguio-un-parque-para-chamberi.
Otero Maldonado, Jorge (2021, 14 de septiembre). De interés general, un barrio por un parque: los héroes de barrio que tumbaron uno de los símbolos de la corrupción del PP de Esperanza Aguirre [entrada de blog]. Parque sí en Chamberí. Recuperado el 21 de septiembre de 2021 de: https:/ / www. publico.es/sociedad/interes-general-barrio-parqueheroes-barrio-tumbaron-simbolos-corrupcion-pp-esperanza-aguirre.html.

Parque Sí en Chamberí y Sánchez, Miguel Ángel (2021). De interés general (un barrio por un parque). Recuperado el 16 de marzo de 2021 de: https:/ / www.youtube.com/watch?v=9yRVduesKpI.

Parque Sí en Chamberí (2017, 19 de enero). Documentos elaborados en el taller de participación vecinal "Por fin si, parque en Chamberi”. Recuperado el 16 de marzo de 2021, de: https://www.parquesienchamberi. org/archives/651.

Parque Sí en Chamberí, AA VV El Organillo de Chamberí, Ecologistas en Acción, asociaciones y AMPAS de Chamberí, con la colaboración de Basurama (organizadores) (2007). Ideas alternativas al campo de golf en Chamberí, Taller de Participación Ciudadana. Recuperado el 16 de marzo de 2021 de: https://www.parquesienchamberi.org/archives/ date $/ 2007 / 04$

Tribunal Superior de Justicia de Madrid (2016, 26 de julio). Sentencia $n^{\circ}$ 580/2016. Madrid: Tribunal Superior de Justicia de Madrid. Recuperado el 16 de marzo de 2021 de: https:/ / www.parquesienchamberi.org/wp-content/uploads /2016/09/201607-26-SENTENCIA-PLAN-ESPECIAL-parquesi-1. pdf.

Warner Bross ITVP España (2007, 10 de febrero). Chamberi Parque SÍ Golf NO. Caiga Quien Caiga. Recuperado el 16 de marzo de 2021 de: https:/ /www. parquesienchamberi.org/archives/603.

Arce Sainz, Mercedes (2021). La Asociación Parque Sí en Chamberí y la participación de los vecinos en la definición y uso del Parque sobre el Tercer Depósito del CYII en Chamberí. Hábitat y Sociedad, 14, 327-337.

<https://doi.org/10.12795/HabitatySociedad.2021.i14.17> 


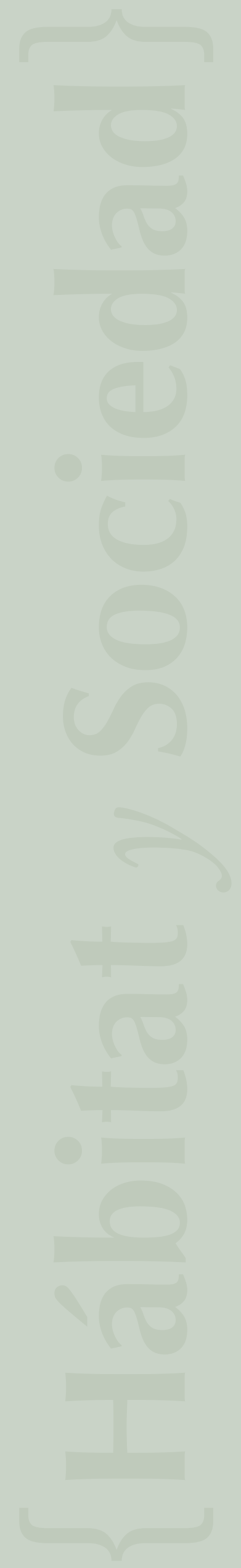

